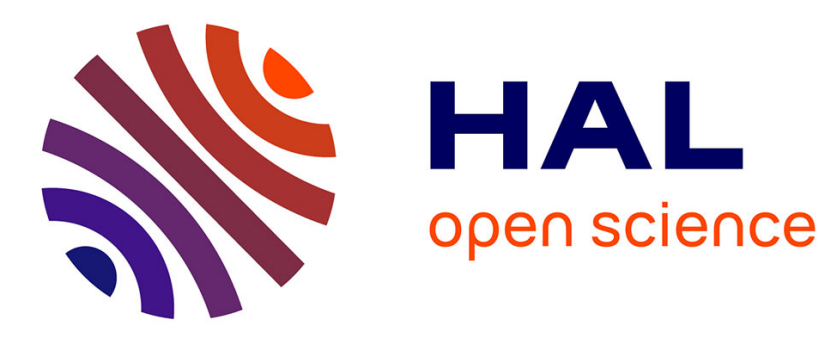

\title{
Softening Gcc and Regular with preferences
}

Jean-Philippe Metivier, Patrice Boizumault, Samir Loudni

\section{To cite this version:}

Jean-Philippe Metivier, Patrice Boizumault, Samir Loudni. Softening Gcc and Regular with preferences. 24th annual ACM Symposium on Applied Computing (SAC'09), Mar 2009, University of Hawaii at Manoa, USA, United States. pp.1392-1396. hal-01015040

\section{HAL Id: hal-01015040 https://hal.science/hal-01015040}

Submitted on 25 Jun 2014

HAL is a multi-disciplinary open access archive for the deposit and dissemination of scientific research documents, whether they are published or not. The documents may come from teaching and research institutions in France or abroad, or from public or private research centers.
L'archive ouverte pluridisciplinaire HAL, est destinée au dépôt et à la diffusion de documents scientifiques de niveau recherche, publiés ou non, émanant des établissements d'enseignement et de recherche français ou étrangers, des laboratoires publics ou privés. 


\title{
Softening Gcc and Regular with preferences
}

\author{
Jean-Philippe Métivier Patrice Boizumault \\ GREYC (UMR 6072) - University of Caen \\ Campus Côte de Nacre \\ Bd du Maréchal juin - BP 5186 \\ 14032 Caen CEDEX - France \\ \{jmetivie,patrice.boizumault\}@info.unicaen.fr loudni@iutc3.unicaen.fr \\ Samir Loudni
}

\begin{abstract}
In this paper, we present the soft global constraints $\Sigma$-gcc and $\Sigma$-regular which are the soft versions with preferences of well-known global constraints Gcc and Regular. For each of them, we introduce a new violation based semantic which takes into account preferences and propose algorithms to enforce hyperarc consistency in polynomial time, making use of flow theory.
\end{abstract}

\section{INTRODUCTION}

Many real-life problems are over-constrained since there exists no solution satisfying all the constraints. In this situation, it is natural to allow certain constraints, the soft ones, to be violated.

Soft versions of some well-known global constraints, such AllDifferent, Gcc and Regular have been recently introduced $[8,9]$. But these soft global constraints do not take into account preferences.

When expressing preferences, each constraint has a weight reflecting its importance (amount of violation or "cost to pay" if the constraint is not satisfied). For an instantiation, the amount of violation is the sum of the weights of all unsatisfied constraints. A first softening of a global constraint with preferences has been proposed for AllDifferent in [3].

In this paper, we present the soft global constraints $\Sigma-$ gcc and $\Sigma$-regular which are the soft versions, with preferences, of the global constraints Gcc and Regular. For each of these two constraints, we introduce a new violation based semantic which takes into account preferences between violations and propose algorithms to enforce hyperarc consistency in polynomial time, making use of flow theory.

In Section 2, we introduce our decomposition based semantic of violation for $\Sigma$-gcc and show how hyperarc consistency can be enforced in polynomial time. In Section 3, we present our value based semantic of violation for $\Sigma$-regular, and show how hyperarc consistency can be enforced in polynomial time thanks to path computations in directed graph. Finally, we conclude and draw some future works.

Permission to make digital or hard copies of all or part of this work for personal or classroom use is granted without fee provided that copies are not made or distributed for profit or commercial advantage and that copies bear this notice and the full citation on the first page. To copy otherwise, to republish, to post on servers or to redistribute to lists, requires prior specific permission and/or a fee.

SAC'09 March 8-12, 2009, Honolulu, Hawaii,U.S.A.

Copyright 2009 ACM 978-1-60558-166-8/09/03 ...\$5.00.

\section{SOFT GLOBAL CONSTRAINT $\Sigma$-GCC}

\subsection{The Global Cardinality Constraint}

JC. Régin has introduced in [5] the Global Cardinality Constraint (Gcc). This constraint defined on a set of $n$ variables specifies, for each value in the union of theirs domains (Doms), bounds on the maximal $\left(u_{j}\right)$ and minimal $\left(l_{j}\right)$ number of times values $v_{j}$ can occur in a solution.

DeFINITION 1. (Gcc, [5]) Let $\mathcal{X}$ be a set of $n$ variables and $D_{i}$ be the domain of the variable $X_{i}$, let Doms $=\cup_{i \in \mathcal{X}} D_{i}$. Let $l_{j}, u_{j} \in \mathbb{N}$ with $l_{j} \leqslant u_{j}$ for all $v_{j} \in$ Doms.

$$
\begin{array}{r}
\operatorname{Gcc}(\mathcal{X}, l, u)=\left\{\left(v_{1}, \ldots, v_{n}\right) \in D_{1} \times \ldots \times D_{n}, \text { s.t. } \forall v_{j} \in D o m s,\right. \\
\left.l_{j} \leqslant\left|\left\{X_{i} \in \mathcal{X} \mid X_{i}=v_{j}\right\}\right| \leqslant u_{j}\right\}
\end{array}
$$

THEOREM 1 ([5]). $\operatorname{Gcc}(\mathcal{X}, l, u)$ is hyperarc consistent iff there exits a feasible $s-t$ flow of value $n$ (where $n=|\mathcal{X}|$ ) in the network $\mathcal{N}=\{\mathcal{V}, \mathcal{A}\}$ with:

$$
\begin{aligned}
& \mathcal{V}=\mathcal{X} \cup \text { Doms } \cup\{s, t\} \\
& \mathcal{A}=\mathcal{A}_{s} \cup \mathcal{A}_{\mathcal{X}} \cup \mathcal{A}_{t} \\
& \mathcal{A}_{s}=\left\{\left(s, X_{i}\right) \mid i \in\{1, \ldots, n\}\right\}, \\
& \mathcal{A}_{\mathcal{X}}=\left\{\left(X_{i}, v_{j}\right) \mid v_{j} \in D_{i}, i \in\{1, \ldots, n\}\right\}, \\
& \mathcal{A}_{t}=\left\{\left(v_{j}, t\right) \mid v_{j} \in \text { Doms }\right\} \\
& \text { demand and capacity functions: } \\
& \forall a \in \mathcal{A}_{s}, d(a)=1 \text { and } c(a)=1 \\
& \forall a \in \mathcal{A}_{\mathcal{X}}, d(a)=0 \text { and } c(a)=1 \\
& \forall a \in \mathcal{A}_{t}, d(a)=l_{j} \text { and } c(a)=u_{j}
\end{aligned}
$$

Consistency-check can be performed in $\mathrm{O}(n \times m)$ (where $m=|\mathcal{A}|$ ). To maintain hyperarc consistency, all arcs (and then values) which do not belong to any feasible flow are removed thanks to computation of strongly connected components in $\mathrm{O}(n+m)$ (see [7] for more details).

Example 1. Consider the following CSP:

$$
D_{1}=D_{2}=\{1,2\}, D_{3}=\{1\}, D_{4}=\{2\} \text {, and } D_{5}=\{2,3\}
$$$$
\operatorname{Gcc}\left(\left[X_{1}, X_{2}, X_{3}, X_{4}, X_{5}\right],[1,1,0],[2,2,1]\right) \text {. }
$$

The network associated to this Gcc constraint is described in Figure 1. $(1,2,1,2,3)$ and $(2,1,1,2,3)$ are the two solutions of this CSP.

\subsection{Decomposition Based Semantic for $\Sigma$-GCC}

\subsubsection{Gec as a meta-constraint}

Gcc can be considered as a meta-constraint and decomposed into a set of atleast constraints enforcing the lower bound for each $v_{j} \in$ Doms, and a set of atmost constraints enforcing the upper bound for each $v_{j} \in$ Doms. 


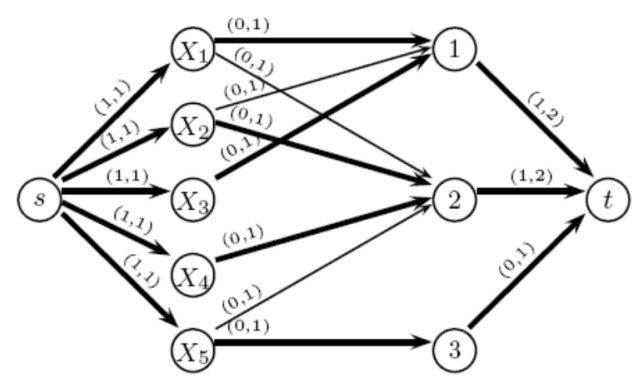

Figure 1: Network representation for the Gcc constraint presented in Example 1, bold arcs represent a feasible flow of value 5 .

Definition 2 ([2]). Let $v_{j} \in$ Doms, let $l_{j}$ and $u_{j}$ their lower and upper bounds,

$$
\begin{aligned}
& \operatorname{atleast}\left(\mathcal{X}, v_{j}, l_{j}\right) \equiv\left|\left\{X_{i} \in \mathcal{X} \mid X_{i}=v_{j}\right\}\right| \geqslant l_{j} \\
& \operatorname{atmost}\left(\mathcal{X}, v_{j}, u_{j}\right) \equiv\left|\left\{X_{i} \in \mathcal{X} \mid X_{i}=v_{j}\right\}\right| \leqslant u_{j}
\end{aligned}
$$

Then, Gcc can be defined as follows:

$$
\operatorname{Gcc}(\mathcal{X}, l, u) \equiv \bigwedge_{v_{j} \in D o m s}\left(\operatorname{atleast}\left(\mathcal{X}, v_{j}, l_{j}\right) \wedge \operatorname{atmost}\left(\mathcal{X}, v_{j}, u_{j}\right)\right)
$$

\subsubsection{Decomposition Based Semantic}

To each value $v_{j} \in$ Doms, we associate a shortage function $\left(s\left(\mathcal{X}, v_{j}\right)\right)$ measuring the number of missing assignments of $v_{j}$ to satisfy atleast $\left(\mathcal{X}, v_{j}, l_{j}\right)$, and an excess function $\left(e\left(\mathcal{X}, v_{j}\right)\right)$ measuring the number of assignments of $v_{j}$ in excess to satisfy atmost $\left(\mathcal{X}, v_{j}, u_{j}\right)[9]$ :

$$
\begin{aligned}
& s\left(\mathcal{X}, v_{j}\right)=\max \left(0, l_{j}-\left|\left\{X_{i} \mid X_{i}=v_{j}\right\}\right|\right) \\
& e\left(\mathcal{X}, v_{j}\right)=\max \left(0,\left|\left\{X_{i} \mid X_{i}=v_{j}\right\}\right|-u_{j}\right)
\end{aligned}
$$

To express preferences on the minimal/maximal requirements on each value $v_{j}$, we associate to each $l_{j}$ (resp. $u_{j}$ ) a weight $\varphi_{j}^{\text {atleast }}$ (resp. $\varphi_{j}^{\text {atmost }}$ ) denoting the amount of violation to pay if the requirement is not satisfied. We can define the violation cost of the constraint atleast $\left(\mathcal{X}, v_{j}, l_{j}\right)$ as follows:

$$
\text { weight }\left(\operatorname{atleast}\left(\mathcal{X}, v_{j}, l_{j}\right)\right)=\varphi_{j}^{\text {atleast }} \times s\left(\mathcal{X}, v_{j}\right)
$$

And respectively for the constraint $\operatorname{atmost}\left(\mathcal{X}, v_{j}, u_{j}\right)$ :

$$
\text { weight }\left(\operatorname{atmost}\left(\mathcal{X}, v_{j}, u_{j}\right)\right)=\varphi_{j}^{\text {atmost }} \times e\left(\mathcal{X}, v_{j}\right)
$$

Let $C_{d e c}$ be the decomposition of a Gcc constraint into a set of atleast and atmost constraints. The decomposition based semantic $\mu_{d e c}$ computes the weighted sum of $\operatorname{atleast}\left(\mathcal{X}, v_{j}, l_{j}\right)$ and atmost $\left(\mathcal{X}, v_{j}, u_{j}\right)$ constraints in $C_{d e c}$ that are violated.

\section{Definition 3. (Decomposition Based Violation Measure)}

$\mu_{\text {dec }}(\mathcal{X})=\sum_{v_{j} \in \text { Doms }} \varphi_{j}^{\text {atleast }} \times s\left(\mathcal{X}, v_{j}\right)+\varphi_{j}^{\text {atmost }} \times e\left(\mathcal{X}, v_{j}\right)$

Definition 4. (Consistency of $\Sigma-g c c$ ) Let $z$ be a cost variable with domain $D_{z}, \Sigma-g c c\left(\mathcal{X}, l, u, z, \mu_{\text {dec }}\right)$ is hyperarc consistent iff there exists a complete instanciation $\mathcal{A}$ s.t. $\mu_{\text {dec }}(\mathcal{A}) \leqslant \max \left(D_{z}\right)$.

\begin{tabular}{|l|l|}
\hline Employee & Shifts \\
\hline Mick & $M$ \\
John & $M$ \\
James & $M, A$ \\
Patty & $M, A$ \\
Julia & $M$ \\
Helen & $A, N$ \\
\hline
\end{tabular}

\begin{tabular}{|l|l|l|l|l|}
\hline Shifts & \multicolumn{2}{|c|}{ Bounds } & \multicolumn{2}{c|}{ Costs } \\
& $l$ & $u$ & $\varphi^{\text {atleast }} \varphi^{\text {atmos }}$ \\
\hline $\mathrm{M}$ & 1 & 2 & 4 & 2 \\
$\mathrm{~A}$ & 3 & 4 & 1 & 2 \\
$\mathrm{~N}$ & 2 & 2 & 3 & 4 \\
\hline
\end{tabular}

Table 1: Availabilities

Table 2: Bounds and costs

\subsubsection{Example}

Consider the following planning problem that consists in building timetables for a group of six employees over a period of a week (more precisely from Monday to Thursday). There are three basic shifts in a day, namely Morning (M), Afternoon (A) and Night (N).

Each shift requires a minimal number of employees to be operational, and a maximal number of employees to be profitable (see Table 2). Each employee provides the set of its availabilities (see Table 1 ).

This problem can be easily modeled as a CSP: to each employee $i$, we associate a variable $X_{i}$ whose domain $D_{i}$ contains the shift requests of employee $i$; bounds on shift requirements can be expressed using a Gcc constraint.

$$
\begin{aligned}
& D_{1}=D_{2}=\{M\}, D_{3}=D_{4}=\{M, A\}, D_{5}=\{M\}, D_{6}=\{A, N\} \\
& \operatorname{Gcc}\left(\left[X_{1}, X_{2}, X_{3}, X_{4}, X_{5}, X_{6}\right],[1,3,2],[2,4,2]\right) .
\end{aligned}
$$

But, shift requests of employees are frequently too restrictive in order to get a solution. The violation of the mini$\mathrm{mal} /$ maximal requirements for a shift may not have the same importance w.r.t. to the profits. In fact, for a Morning shift, it is more important to respect its lower bound $\left(l_{i}\right)$ rather than its upper bound $\left(u_{i}\right)$, since any violation of $l_{i}$ may have a great impact on the other shifts (i.e., the production may be delayed). In contrast, for a Night shift, one prefers to respect its upper bound $\left(u_{i}\right)$ rather than its lower bound because having one more worker could be considered as too expensive. Table 2 summarizes, for each shift, the values of $l_{i}$ and $u_{i}$ and their violation costs.

This over-constrained problem can be now expressed as a CSP with preferences (on shift requirements) using a $\Sigma$-gcc constraint.

$$
\begin{aligned}
& D_{1}=D_{2}=\{M\}, D_{3}=D_{4}=\{M, A\}, D_{5}=\{M\}, D_{6}=\{A, N\} \\
& D_{z}=[0 . .6], \Sigma-\operatorname{gcc}\left(\left[X_{1}, X_{2}, X_{3}, X_{4}, X_{5}, X_{6}\right],[1,3,2],[2,4,2], z, \mu_{d e c}\right) .
\end{aligned}
$$

The solution $(M, M, A, A, M, N)$, whose violation cost is equal to $1 \times \varphi_{M}^{\text {atmost }}+1 \times \varphi_{A}^{\text {atleast }}+1 \times \varphi_{N}^{\text {atleast }}=2+4=6$, satisfies the $\Sigma$-gcc as its cost is less or equal to $\max \left(D_{z}\right)=6$. Figure 2 shows the network associated to this example.

\subsection{Network Representation for $\Sigma$-GCC}

As for Gcc, $\Sigma$-gcc can be modeled by a network. From the initial network $\mathcal{N}$ associated to Gcc (see Section 2.1), WJ. van Hoeve and $a l$. have defined the network $\mathcal{N}_{\text {soft }}$ associated to soft-gcc [9]. Violation arcs are added in order to model shortage and excess functions:

- Shortage violation arcs, $\mathcal{A}_{\text {shortage }}=\left\{\left(s, v_{j}\right) \mid v_{j} \in\right.$ Doms $\}$, are used to model atleast $\left(\mathcal{X}, v_{j}, l_{j}\right)$ constraints. For each shortage violation arc $a=\left(s, v_{j}\right)$, its demand $d(a)=0$, its capacity $c(a)=l_{j}$, and its weight $w(a)=1$.

- Excess violations arcs, $\mathcal{A}_{\text {excess }}=\left\{\left(v_{j}, t\right) \mid v_{j} \in\right.$ Doms $\}$, are used to model atmost $\left(\mathcal{X}, v_{j}, u_{j}\right)$ constraints. For 


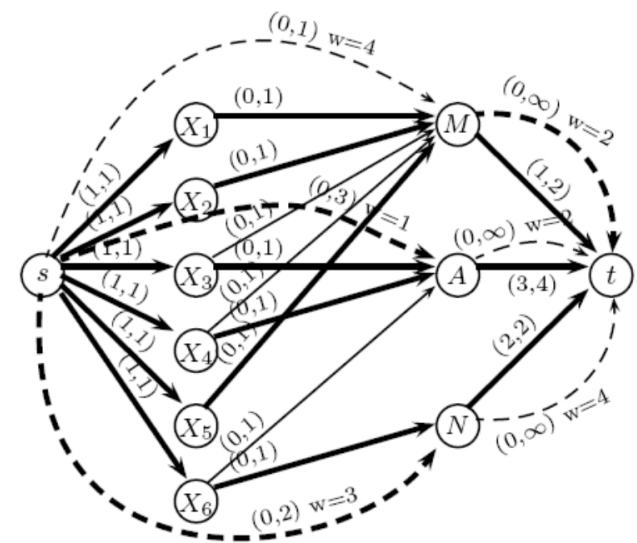

Figure 2: Network representation for the $\Sigma$-gcc presented in section 2.2 .3 , bold arcs represent a maximal flow of weight 6 .

each excess violation arc $a=\left(v_{j}, t\right)$, its demand $d(a)=0$, its capacity $c(a)=\infty$, and its weight $w(a)=1$.

Now, we can define the network $\mathcal{N}_{\Sigma}$ associated to a $\Sigma$-gcc constraint. For the decomposition based semantic introduced in Section 2.2.2, the cost associated to the violation of a lower bound $\left(l_{i}\right)$ is not necessarily the same to the one associated to the violation of an upper bound $\left(u_{i}\right)$ :

- Let $v_{j} \in$ Doms, and $\varphi_{j}^{\text {atleast }}$ be the weight associated to the violation of the lower bound $l_{j}$ for $v_{j}$. For each shortage violation arc $a=\left(s, v_{j}\right)$, its demand $d(a)=0$, its capacity $c(a)=l_{j}$, and its weight $w(a)=\varphi_{j}^{\text {atleast }}$.

- Let $v_{j} \in$ Doms, and $\varphi_{j}^{\text {atmost }}$ be the weight associated to the violation of the upper bound $u_{j}$ for $v_{j}$. For each excess violation arc $a=\left(v_{j}, t\right)$, its demand $d(a)=0$, its capacity $c(a)=\infty$, and its weight $w(a)=\varphi_{j}^{a t m o s t}$.

\subsection{Consistency-Check}

Let $z$ be a cost variable that represents the allowed amount of violation of a $\Sigma$-gcc constraint. To check the consistency of a $\Sigma$-gcc constraint we use the following corollary:

Corollary 1. The constraint $\Sigma-g c c\left(\mathcal{X}, l, u, z, \mu_{d e c}\right)$ is hyperarc consistent if and only if there exists an integer $s-t$ flow $f$ of value $n$ in $\mathcal{N}_{\Sigma}$ with weight $(f) \leqslant \max \left(D_{z}\right)$.

Proof. To an integer $s-t$ flow $f$ of value $n$ in $\mathcal{N}_{\Sigma}$ we associate the assignment $X_{i}=v_{j}$ for all arcs $a=\left(X_{i}, v_{j}\right) \in \mathcal{A}_{\mathcal{X}}$ with $f(a)=1$. By construction, the cost function measures the decomposition based violation cost.

Consistency-Check can be performed thanks to a Ford \& Fulkerson algorithm in $O\left(\max \left(n, \sum_{v_{j} \in \text { Doms }} l_{j}\right) \times(m+n \times\right.$ $\log (n)))[1]$.

\subsection{Maintaining hyperarc consistency}

To maintain hyperarc consistency, we use the following corollary:

COROLLARY 2. The constraint $\Sigma-g c c$ is hyperarc consistent if and only if for every arc $a \in \mathcal{A}_{\mathcal{X}}$ there exists an integer $s-t$ flow $f$ of value $\max \left(n, \sum_{v_{j} \in \text { Doms }} l_{j}\right)$ in $\mathcal{N}_{\Sigma}$ with $f(a)=1$ and weight $(f) \leqslant \max \left(D_{z}\right)$.
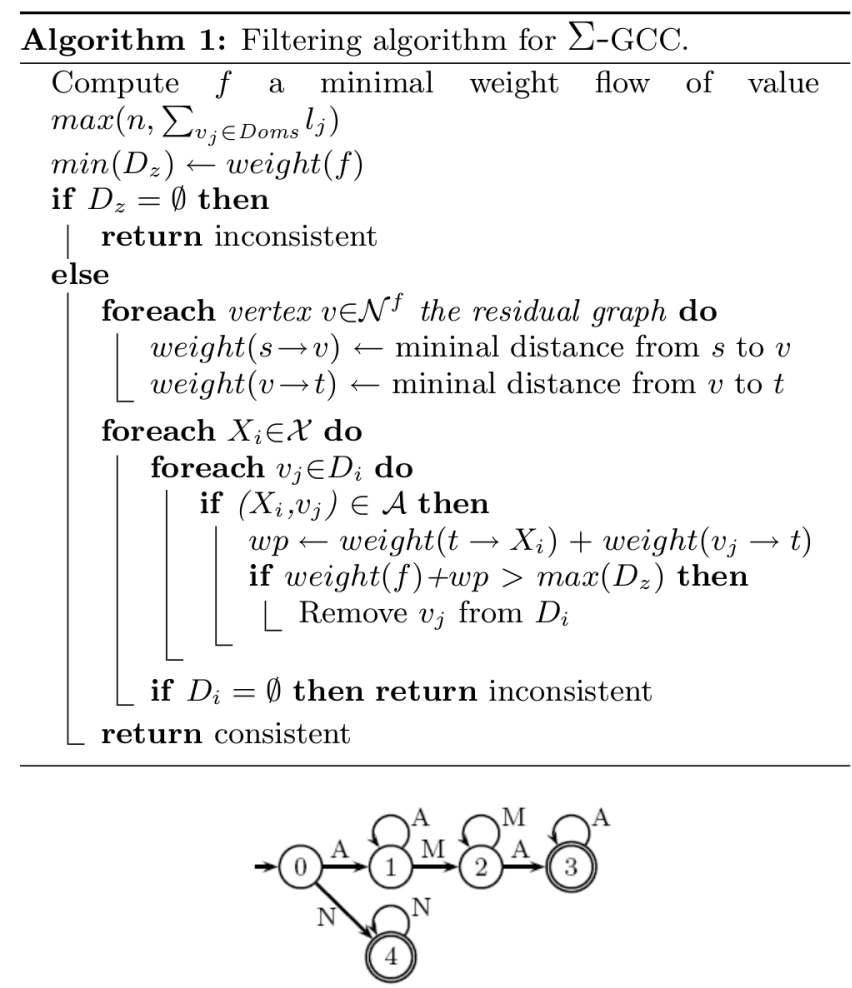

Figure 3: DFA associated to the timetabling

The $\Sigma$-gcc of Example 2 can be made hyperarc consistent by removing value $\mathrm{M}$ from $D_{3}$ and $D_{4}$ and value A from $D_{6}$.

We can use the same technique as cost-gcc [6] to compute the weight of a flow using a specific arc.

For each arc $\left(X_{i}, v_{j}\right)$, we compute the weight of rerouting the flow in order to use this arc thanks to the search of the minimal weighted cycle $\left(v_{j} \rightarrow t \rightarrow s \rightarrow X_{i} \rightarrow v_{j}\right)$. Then, we add this weight to the initial flow. If this sum is greater than $\max \left(D_{z}\right)$, we have to remove the value $v_{j}$ from $D_{i}$ (see Algorithm 1).

Complexity of the filtering is $O((n+d) \times(m+n \times \log (n)))$.

\section{A SOFT REGULAR: $\Sigma$-REGULAR}

\subsection{The Regular Constraint}

\subsubsection{Definition and Example}

Let $\mathcal{M}=\left(Q, \Sigma, \delta, q_{0}, F\right)$ be a deterministic finite automaton (DFA) where Q a finite set of states, $\Sigma$ an alphabet, $\delta$ a transition function defined on $Q \times \Sigma \rightarrow Q, q_{0}$ the initial state and $F \subseteq Q$ the set of final states. Given an input word, the automaton starts in the initial state $q_{0}$ and processes the word one symbol at a time, applying the transition function $\delta$ at each step. The word is accepted if and only if the last state reached belongs to $F$. All words accepted by a DFA belong to the language recognized by the DFA noted $L(\mathcal{M})$.

For the automaton described in Figure 3, words $A A M A$ and $N N N$ belong to $L(\mathcal{M})$ but it is not the case for $N N A N$.

The Regular constraint states that a word, represented by a sequence of $n$ variables $X_{1}, X_{2}, \ldots, X_{n}$, has to be accepted by a given DFA.

DeFinition 5. (Regular [4]) Let $\mathcal{M}=\left(Q, \Sigma, \delta, q_{0}, F\right)$ be 
a DFA and $\mathcal{X}=X_{1}, X_{2}, \ldots, X_{n}$ be a sequence of $n$ variables with respective domains $D_{1}, D_{2}, \ldots, D_{n} \subset \Sigma$. Then

$\operatorname{Regular}(\mathcal{X}, \mathcal{M})=\left\{\left(v_{1}, \ldots, v_{n}\right) \in D_{1} \times \ldots \times D_{n} \mid\left(v_{1}, \ldots, v_{n}\right) \in L(\mathcal{M})\right\}$.

\subsubsection{Graph associated to a Regular constraint}

Regular can be modeled with a directed graph.

Theorem 2. ([4]) Let $\mathcal{M}=\left(Q, \Sigma, \delta, q_{0}, F\right)$ be a DFA. $A$ constraint Regular $(\mathcal{X}, \mathcal{M})$ is hyperarc consistent iff there exists an $s-t$ path in the graph $\mathcal{G}=\{\mathcal{V}, \mathcal{A}\}$ defined as follows:

$$
\begin{aligned}
& \mathcal{V}=\mathcal{V}_{1} \cup \mathcal{V}_{2} \cup \cdots \cup \mathcal{V}_{n} \cup\{s, t\} \\
& \text { where } \mathcal{V}_{i}=\left\{q_{k}^{i} \mid q_{k} \in Q\right\} \text { for } i=1, \ldots, n+1 \\
& \text { and } \mathcal{A}=\mathcal{A}_{s} \cup \mathcal{A}_{1} \cup \mathcal{A}_{2} \cup \cdots \cup \mathcal{A}_{n} \cup \mathcal{A}_{t} \\
& \text { where } \\
& \mathcal{A}_{s}=\left\{\left(s, q_{0}\right)\right\} \\
& \mathcal{A}_{i}=\left\{\left(q_{k}^{i}, q_{l}^{i+1}, v_{j}\right) \mid v_{j} \in D_{i}, \delta\left(q_{k}, v_{j}\right)=q_{l}\right\} \text { for } i=1, \ldots, n \\
& \left.\mathcal{A}_{t}=\left\{\left(q_{k}^{n+1}, t\right) \mid q_{k} \in F\right)\right\}
\end{aligned}
$$

\subsubsection{Consistency-Check}

The Consistency-Check can be efficiently performed by computing an $s-t$ path thanks to a breadth-first search in $O(m)$, where $m$ is the number of arcs in $\mathcal{G}$ (with $m \leqslant n \times 1$ $Q|\times| \Sigma \mid)$.

\subsubsection{Filtering}

Filtering can be performed in two steps [4]:

1. For each vertex (different from the target $t$ ) with no outgoing arc, all incoming arcs are removed. For each vertex (different from the sink $s$ ) with no incoming arc, all outgoing arcs are removed.

2. If there exists no $\operatorname{arc}\left(q_{k}^{i}, q_{l}^{i+1}, v_{j}\right)$ between $q_{k}^{i}$ and $q_{l}^{i+1}$, then value $v_{j}$ can be removed from domain $D_{i}$.

So, filtering consists in checking each arc and it can be performed in $O(m)$.

\subsection{Distance Based Semantic for $\Sigma$-REGULAR}

In this section, we introduce a new value based semantic taking into account the distance between expected and unexpected values for a transition.

Definition 6. (Expected value) Value $v_{j} \in \Sigma$ is said to be expected for a couple of states $\left(q_{k}, q_{l}\right)$ if and only if there exists a transition from state $q_{k}$ to state $q_{l}$ using symbol $v_{j}$. $E V\left(q_{k}, q_{l}\right)=\left\{v \in \Sigma \mid \delta\left(q_{k}, v\right)=q_{l}\right\}$ will denote the set of expected values for $\left(q_{k}, q_{l}\right)$.

Definition 7. (Distance) Let $\left(q_{k}, q_{l}\right)$ be a couple of states, value $v_{j} \in E V\left(q_{k}, q_{l}\right)$ and value $v_{i} \in \Sigma$.

- distance $\left(v_{j}, v_{i}\right)=0$ if $v_{i} \in E V\left(q_{k}, q_{l}\right)$

- $\operatorname{distance}\left(v_{j}, v_{i}\right)=\varphi_{i, j}$ if $v_{i} \in \Sigma \backslash E V\left(q_{k}, q_{l}\right)$

where each $\varphi_{i, j}$ corresponds to the cost for having performed a transition from $q_{k}$ to $q_{l}$ using an unexpected value $v_{i}$ instead of expected value $v_{j}$.

Between two words, the value based distance, $V\left(W_{a}, W_{b}\right)$, is the sum of the distances between their corresponding symbols. The value based distance between a word and a language is the minimal value based distance between the word and any word of the language.

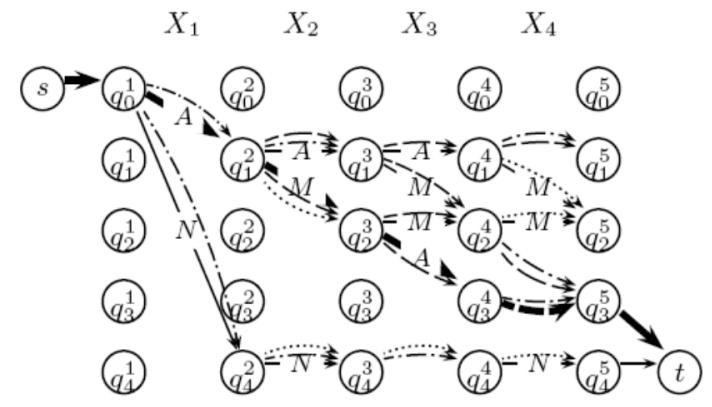

Figure 4: Graph for $\Sigma$-regular $\left(\mathcal{X}, \mathcal{M}, \mu_{\text {val }}, z\right)$ with $\mathcal{M}$ the DFA presented in Figure 3. Dashed arcs represent a $M \leftrightarrow A$ move, Dotted one a $M \leftrightarrow N$ move and mixed ones a $A \leftrightarrow N$ move. Bold arcs represent the solution $(A, M, A, M)$ of cost 1 .

Definition 8. (Value Based Semantic Measure) For a $\Sigma-\operatorname{regular}\left(\mathcal{X}, \mathcal{M}, z, \mu_{\text {val }}\right)$ constraint, the value based violation measure is defined as:

$$
\mu_{\text {val }}(\mathcal{X})=\min _{W \in L(\mathcal{M})} V(\mathcal{X}, W)
$$

Definition 9. (Consistency of $\Sigma$-regular) Let $z$ be a cost variable with domain $D_{z}, \Sigma$-regular $\left(\mathcal{X}, \mathcal{M}, z, \mu_{\text {val }}\right)$ is consistent iff there exists a complete instiation $\mathcal{A}$ such that $\mu_{\text {val }}(\mathcal{A}) \leqslant \max \left(D_{z}\right)$.

\subsection{Graph Representation for $\Sigma$-regular}

A $\Sigma$-regular constraint can be modeled as a graph $\mathcal{G}_{\Sigma}$. Let $\mathcal{G}$ be the valued graph associated to a Regular constraint (see 3.1.2). For each layer $i$, and each couple of states $\left(q_{k}, q_{l}\right)$ linked by at least one transition, $\mathcal{A}_{i, k, l}=\left\{\left(q_{k}^{i}, q_{l}^{i+1}, v\right)\right.$ | $\left.v \in D_{i} \backslash E V\left(q_{k}^{i}, q_{l}^{i+1}\right)\right\}$ denotes the set of violation arcs associated to unexpected values for $\left(q_{k}, q_{l}\right)$. Each violation $\operatorname{arc}\left(q_{k}^{i}, q_{l}^{i+1}, v\right)$ of each $\mathcal{A}_{i, k, l}$ is added to $\mathcal{G}$. Let $v_{j}$ in $E V\left(q_{k}^{i}, q_{l}^{i+1}\right)$; the valuation of $\left(q_{k}^{i}, q_{l}^{i+1}, v\right)$ is distance $\left(v_{j}, v\right)$, in order that transitions performed using unexpected values induce extra-cost.

Corollary 3. Let $z$ be a cost variable with domain $D_{z}$, a constraint $\Sigma$-regular $\left(\mathcal{X}, \mathcal{M}, z, \mu_{\text {val }}\right)$ is hyperarc consistent if and only if there exists an $s-t$ path $p$ in $\mathcal{G}_{\Sigma}$ such that weight $(p) \leqslant \max \left(D_{z}\right)$.

Proof. To an $s-t$ path in $\mathcal{G}_{\Sigma}$, we associate, to each variable $X_{i}$ a value $v_{j}$ for all arc $\left(q_{k}^{i}, q_{l}^{i+1}\right)$ using the value $v_{j}$ such that $\left(q_{k}^{i}, q_{l}^{i+1}, v_{j}\right)$ belongs to $p$. By construction, the weight of $p$ corresponds exactly to the value based measure of violation.

Consider Figure 4 where $\mathcal{M}$ is the DFA depicted in Figure $3, \mathcal{X}=\left\{X_{1}, X_{2}, X_{3}, X_{4}\right\}, D_{1}=\{A, N\}, D_{2}=\{M, A, N\}$, $D_{3}=\{M, A\}, D_{4}=\{M, N\}$. Violation arcs are added in dashed, dotted and mixed.

\subsection{A timetabling example}

Consider again the previous timetabling problem. The consecutive shifts of an employee have to respect the following rules:

- Rule 1: If an $\mathrm{N}$ shift is assigned to an employee for a particular day, this shift must be assigned to this employee for all the week. 


\begin{tabular}{|c|ccc|}
\hline Cost & $\mathrm{M}$ & $\mathrm{A}$ & $\mathrm{N}$ \\
\hline $\mathrm{M}$ & - & 1 & 4 \\
$\mathrm{~A}$ & 1 & - & 2 \\
$\mathrm{~N}$ & 4 & 2 & - \\
\hline
\end{tabular}

Table 3: Shift moves and their costs.

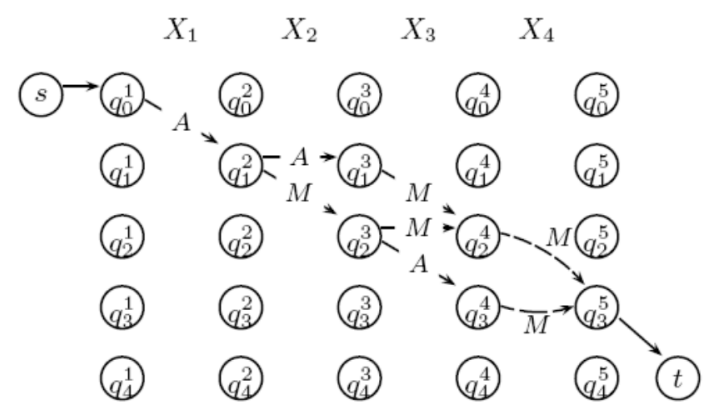

Figure 5: $\quad$ Filtered Network for $\Sigma$-regular $\left(\mathcal{X}, \mathcal{M}, \mu_{\text {val }}, z\right)$ in Figure 4 with the filtered domains $D_{1}=\{A\}, D_{2}=D_{3}=\{A, M\}$ and $D_{4}=\{M\}$.

- Rule 2: An employee having an A shift on Monday must have at least an $M$ shift over the week. Moreover, an A shift must be assigned to this employee on Thursday.

These planning rules can be modeled as a DFA (see Figure 3) and can be expressed using a Regular constraint. The language recognized by the DFA is $N N^{\star}$ for Rule 1 and $A A^{\star} M M^{\star} A A^{\star}$ for Rule 2.

As shift requests make the problem over-constrained, we have to soften the Regular constraint with preferences (i.e. $\Sigma$-regular) in order to get an acceptable solution. For an employee and a particular day, moving from one shift to another one will be allowed, but it will introduce a cost/weight relative to the non-respect of the two planning rules. For instance, moving an employee from $\mathrm{M}$ to $\mathrm{N}$ has a high cost compared to moving an employee from A to N. Moving an employee from $\mathrm{M}$ to $\mathrm{A}$ induces a low cost. Table 3 summarizes the cost of each move.

$\Sigma$-regular enables to express and quantify the violation of planning rules. Figure 4 shows its associated network.

\subsection{Maintaining Hyperarc consistency}

Filtering can be performed in three steps (see Figure 5):

- For each vertex with no outgoing arc, all incoming arcs are removed. For each vertex with no incoming arc, all outgoing arcs are removed.

- For each remaining arc $a$ in the network, if there does not exist an $s-t$ path using $a$ with a weight lower or equal to $\max \left(D_{z}\right)$, then arc $a$ is removed.

- As for the second step of Regular's filtering algorithm, for all $k$ and $l$ if a value $v_{j}$ does not appear in at least one arc between $q_{k}^{i}$ and $q_{l}^{i+1}$, then $v_{j}$ can be removed from domain $D_{i}$.

For an acyclic graph, computing a shortest path having a particular arc can be performed in $O(m)$. If we use the same path computation as cost-gcc [6], filtering can be performed in $\mathrm{O}(\mathrm{m})$.

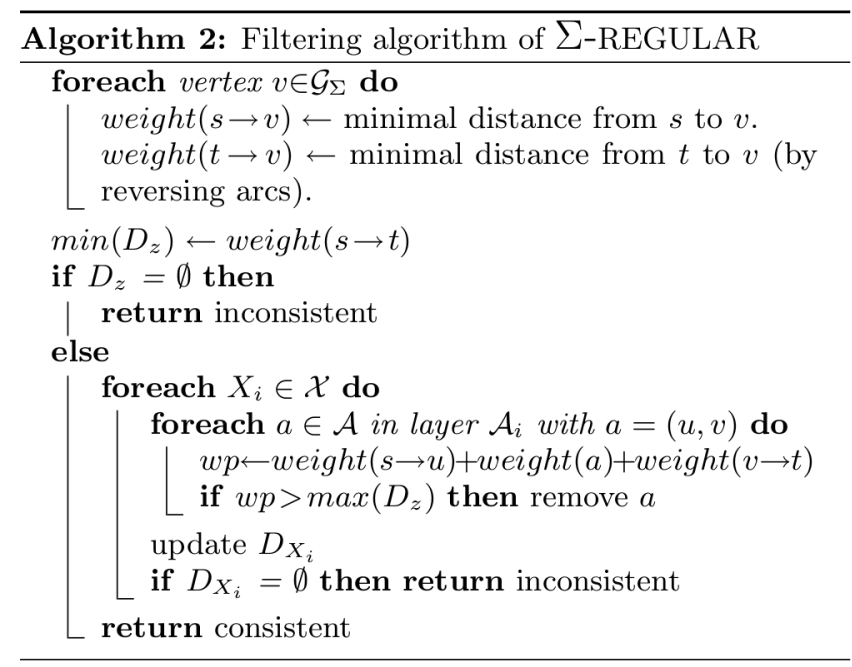

\section{CONCLUSIONS}

In this paper we have presented the soft global constraints $\Sigma$-gcc and $\Sigma$-regular which are the soft versions with preferences of the global constraints Gcc and Regular. For each of these two constraints, we have introduced a new violation semantic and proposed algorithms to enforce hyper-arc consistency in polynomial time, thanks to a network modelisation and flow algorithms.

In future works we want to improved $\Sigma$-gcc by using the matching based modeling proposed by A. Zanarini and al. in [11]. Many problems involve several global constraints which share a subset of variables. In this case, we want to study whether these interactions between global constraints can be used to compute better lower bounds.

\section{REFERENCES}

[1] L.R. Ford and D.R. Fulkerson. Flow in Networks. Princeton University Press, 1962.

[2] P. Van Hentenryck, H. Simonis, and M. Dincbas. Constraint satisfaction using constraint logic programming. Artif. Intell., 58(1-3):113-159, 1992.

[3] J.P. Métivier, P. Boizumault, and S. Loudni. $\Sigma$-AllDifferent: Softening alldifferent in weighted csps. In ICTAI (1), pages 223-230. IEEE Computer Society, 2007.

[4] G. Pesant. A regular language membership constraint for finite sequences of variables. In Wallace [10], pages 482-495.

[5] J.C. Régin. Generalized arc consistency for global cardinality constraint. In $A A A I / I A A I$, Vol. 1, pages 209-215, 1996.

[6] J.C. Régin. Arc consistency for global cardinality constraints with costs. In J. Jaffar, editor, $C P$, volume LNCS 1713, pages 390-404. Springer, 1999.

[7] R.E. Tarjan. Depth-first search and linear graph algorithms. SIAM J. Comput., 1(2):146-160, 1972.

[8] W.J. van Hoeve. A hyper-arc consistency algorithm for the soft alldifferent constraint. In Wallace [10], pages 679-689.

[9] W.J. van Hoeve, G. Pesant, and L.M. Rousseau. On global warming: Flow-based soft global constraints. J. Heuristics, 12(4-5):347-373, 2006.

[10] M. Wallace, editor. CP 2004, volume LNCS 3258. Springer, 2004.

[11] A. Zanarini, M. Milano, and G. Pesant. Improved algorithm for the soft global cardinality constraint. In J. Christopher Beck and Barbara M. Smith, editors, CPAIOR, volume 3990 of $L N C S$, pages 288-299. Springer, 2006. 